\title{
Aligned carbon nanotube based sensors for strain sensing applications
}

\author{
A. Santos $^{a, *}$, L. Amorim ${ }^{\text {a }}$, J.P. Nunes ${ }^{\text {a }}$, L.A. Rocha ${ }^{\mathrm{b}}$, A.F. Silva ${ }^{\mathrm{b}}$, J.C. Viana ${ }^{\mathrm{a}}$ \\ a IPC/i3N - Institute for Polymers and Composites, University of Minho, Guimarães, Portugal \\ ${ }^{\mathrm{b}}$ CMEMS - Center for MicroElectroMehanical Systems, University of Minho, Guimarães, Portugal
}

\section{A R T I C L E I N F O}

\section{Article history:}

Received 10 September 2018

Received in revised form 21 February 2019

Accepted 21 February 2019

Available online 25 February 2019

\section{Keywords:}

CNT

Sensor

Strain monitoring

Anisotropy

\begin{abstract}
A B S T R A C T
This paper presents an aligned carbon nanotube (CNT)-based strain sensor. Vertical aligned carbon nanotubes (VA-CNT), synthesized by chemical vapour deposition (CVD), were knocked down onto polymeric films, in order to obtain a thin $10 \times 10 \times 0.05 \mathrm{~mm}$ CNT patch. Different polymeric substrates, ADEXepoxy, polyethylene terephthalate (PET) and polyimide (PI) were used. The samples' morphology before and after the knock down process, specifically their alignment, was observed by scanning electron microscopy (SEM). The good quality of the synthesized VA-CNT was assessed by Raman spectroscopy. Furthermore, transmission electron microscopy (TEM) analysis was carried out to determine the average wall number and diameters (inner and outer) of the VA-CNT. A MATLAB software with an adapted Van der Pauw method for anisotropic conductors was developed to determine the electric properties of the obtained samples, which were strained in the transverse $(\mathrm{X})$ and parallel $(\mathrm{Y})$ directions with respect to the CNT alignment. The electric anisotropy, defined as electric resistance ratio between obtained measurements along the $X\left(R_{x x}\right)$ and $Y\left(R_{y y}\right)$-axes, decreases with deformation increment when the sample was strained in the Y-direction, while it increases when strained in the X-direction. Moreover, the obtained Gauge factor values showed a much sensitive response to deformation, i.e., approximately $47 \%$ increase in GF values, when the samples are strained transversely to CNT alignment. These results showed that the piezoresistive CNT/polymeric based sensor produced is suitable for strain sensing applications.
\end{abstract}

(C) 2019 Elsevier B.V. All rights reserved.

\section{Introduction}

In the last decades, carbon nanotubes (CNT) have been studied for several applications, namely aerospace, aeronautic, or microelectronics, among others. CNT structural, electric, mechanical and electromechanical properties made them suitable for strain sensors [1]. Moreover, the anisotropic electric properties, due to the CNT alignment, can be an advantage for sensing in different directions or with different direction sensitivity.

CNT have been used for piezoresistive polymer-based sensors by adopting three main approaches:

(a) as conductive matrix fillers in polymer nanocomposites as thin films [2,3];

(b) as thin bucky paper films [4,5]; and

(c) as aligned forests [6,7].

However, the use of CNT as strain sensors rise a few manufacturing issues that become drawbacks on their widely adoption. Main manufacturing steps of CNT-polymer nanocomposite include

\footnotetext{
* Corresponding author.

E-mail address: b7525@dep.uminho.pt (A. Santos).
}

mechanical mixing and deposition methods, such as filtration, coating and dip casting, among others, which present difficulties in the uniformity of CNT dispersion, in the avoidance of agglomerates and in the process of CNT alignment [8]. CNT bucky paper sensors can be produced by dispersion and deposition methods, such as vacuum filtration (the most common method), drop casting, or hot-press compression, among others, which can also result in a non homogeneous CNT distribution $[4,5,9]$. Vertical-aligned carbon nanotubes (VA-CNT) forests impregnation also shows full impregnation deficiency and uncertainty in maintaining the CNT alignment.

The most common method for VA-CNT growth is via chemical vapour deposition (CVD), which shows high process variability and several decisive factors for the CNT electric properties, such as growth time, temperature, catalytic type and thickness, gas mixture and additional delamination step (hydrogen flow after the growth). Some authors studied the effects of CNT length [10] that can be controlled by CVD growth time, and of the interplay of CNT structure and density [11], which can be controlled by the catalytic thickness, on the VA-CNT anisotropic electric properties. Higher growth time results in longer CNT and lower electric resistance [10]. Support/catalytic interaction and adequate hydrogen pre-treatment time (c.a. 2-5 min) affects CNT structure [12], with higher catalytic thickness resulting in two opposite effects: the electric conductivity 
a)

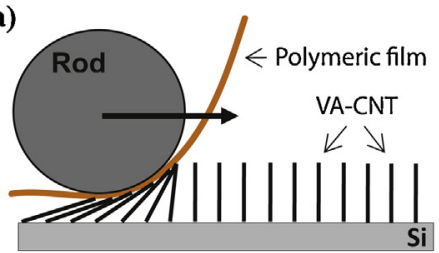

b)

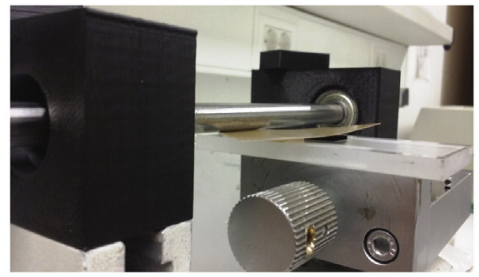

c)

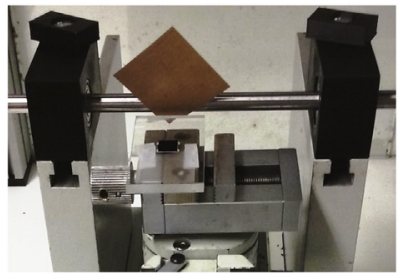

Fig. 1. Schematic (a) and apparatus (b) and (c) of the knock down process.

increases with CNT wall number; however, the electric conductivity also decreases due to the increase in diameter (packing density decreases) with CNT wall number [11]. More recently, knocked down VA-CNT were used as microheaters [13] with possible applications in resin curing of composites materials (out-of-oven curing) and de-icing, and to improve inter-laminar facture toughness on laminated composites [14].

In this work, it is developed a piezoresistive CNT/polymeric film sensor for strain measurements. To avoid the manufacturing issues of typical CNT based sensors, e. g., CNT dispersion and alignment, VA-CNT were used and easily transferred to polymeric films via knock down process to obtain the aligned-CNT based sensor. For this purpose, the CNT were grown via CVD to directly obtain VA-CNT, which were after realigned via knock down process on a polymer substrate. The proposed approach for strain sensing is to take advantage of the electric anisotropic properties of the CNT patch. For this, the electric resistivity in two orthogonal directions were measured during the deformation of different substrate films.

\section{Experimental}

\subsection{CNT synthesis}

VA-CNT were synthetized via CVD, which consists on the CNT chemical growth on a wafer surface inside a horizontal quartz tube with $25 \mathrm{~mm}$ inner diameter, at $750{ }^{\circ} \mathrm{C}$, with a flown gas mixture of ethylene/hydrogen/helium (100/200/55 sccm). The detailed process is described elsewhere [15]. A $10 \times 10 \mathrm{~mm}$ silicon (Si) substrate wafer with $10 \mathrm{~nm}$ thick alumina $\left(\mathrm{Al}_{2} \mathrm{O}_{3}\right) / 1 \mathrm{~nm}$ iron $(\mathrm{Fe})$ catalyst was produced by e-beam vapour deposition, and a photoresistor coating was applied to avoid iron oxidation with time. Then, the wafer was washed with acetone (99.5\%) and alcohol iso-propil (99.8\%) to remove the photoresistor coating. The VA-CNT were grown on the wafer's surface for $24 \mathrm{~min}$, and after a CNT/wafer delamination step was added to facilitate the subsequent CNT knock down process onto the polymeric films.

\subsection{CNT knock down}

For the CNT knock down, an apparatus was developed as shown in Fig. 1. Specifically, a $10 \mathrm{~mm}$ diameter rod was assembled to two 3D printed pieces and applied on top of a micrometric table, which allowed the manual transference of the VA-CNT onto three different polymeric films, namely: polyimide, PI (75 $\mathrm{m}$ Kapton MP film), epoxy (25 $\mu \mathrm{m}$ ADEX epoxy film), and polyethylene terephthalate, PET, which was peeled off of the ADEX epoxy film. The polymeric films were cleaned with acetone and alcohol prior to the CNT knock down process.

\subsection{SEM analysis}

In order to evaluate the CNT height and alignment on the obtained samples before and after the knock down process, scanning electron microscopy (SEM) analysis was carried out in a NanoSEM-200 apparatus from FEI Nova.

\subsection{Raman spectroscopy}

Raman spectroscopy was performed to assess the quality of the synthesized VA-CNT. The spectra was obtained using a HORIBA LabRAM HR Evolution microscope with $532 \mathrm{~nm}$ laser (10x objective, N.A. 0.25), and the CNT wall defects concentrations were evaluated using the integrated intensity ratio, $A_{D} / A_{G}$, of the D and G-band peaks, where $A_{D}$ and $A_{G}$ are the areas of $D$ and G-band peaks, respectively [16].

\subsection{TEM analysis}

In order to experimentally determine the average wall number and diameters (inner and outer) of the VA-CNT, transmission electron microscopy (TEM) analysis was carried out in a JEOL JEM-2100 electron microscope, operating a LaB6 electron gun at $80 \mathrm{kV}$.

\subsection{Electrical ressitivity measurements}

After the knock down process, a silver conductive epoxy adhesive (8330S from MG Chemicals) was used as electrode between the CNT/polymeric film samples and the copper wires, as shown in Fig. 2 (the CNT patch is at the centre of the polymeric film). A MATLAB software was developed to determine the electric properties of the knocked down samples with an adapted Van der Pauw method for anisotropic conductors $[17,18]$. Briefly, the adaptation consists in the conformal transformation from isotropic to anisotropic conductor that results in Eq. (1) through which the $R_{y y, \square} / R_{x x, \square}$ value is obtained [18]:

$$
\frac{a / 2}{b \sqrt{R_{y y, \square} / R_{x x, \square}}}=\frac{\int_{0}^{\pi / 2} \frac{d \varphi}{\sqrt{1-k^{2}(\sin \varphi)^{2}}}}{\int_{0}^{\pi / 2} \frac{d \varphi}{\sqrt{1-(\sin \varphi)^{2}+k^{2}(\sin \varphi)^{2}}}}
$$

where $a$ and $b$ are the CNT's patch square dimensions, which initially is $\mathrm{a}_{0}=\mathrm{b}_{0}=10 \mathrm{~mm}$. These values vary with the deformation of the polymeric film: $a+\Delta l$ in strain direction ( $\Delta l$ is the elongation of the film considering the perfect adhesion between the electrodes and the film), and in the transverse direction $b=b_{0}-v \varepsilon l_{t 0}$ considering the Poisson ratio, $v,(0.34,0.37$ and 0.34 for ADEXepoxy, PET and Polyimide, respectively), where $\varepsilon$ is the mechanical strain and $l_{t 0}$ is the initial width of the film. $R_{y y, \square}$ and $R_{x x, \square}$ are the square resistances in Y-and X-direction, respectively, and $k$ is obtained from Eq. (2).

$\alpha=\frac{V_{D C} / I_{A B}}{V_{B C} / I_{A D}}=\frac{\ln \frac{4 / k}{(1 / k+1)^{2}}}{\ln \frac{(1 / k-1)^{2}}{(1 / k+1)^{2}}}$

Where, as represented in Fig. $3, I_{A B}$ and $I_{A D}$ are the injected currents in $\mathrm{AB}$ and $\mathrm{AD}$ directions, respectively, and $V_{D C}$ and $V_{B C}$ are the voltages measured in $D C$ and $B C$ directions. Then, according to [17], through a simple adaptation of a Van der Pauw equation (Eq. (3)) the value of $R_{y y, \square} R_{x x, \square}$ is obtained:

$e^{-\pi d R_{A B, C D} / \sqrt{\rho_{x} \rho_{y}}}+e^{-\pi d R_{B C, D A} / \sqrt{\rho_{x} \rho_{y}}}=1$ 


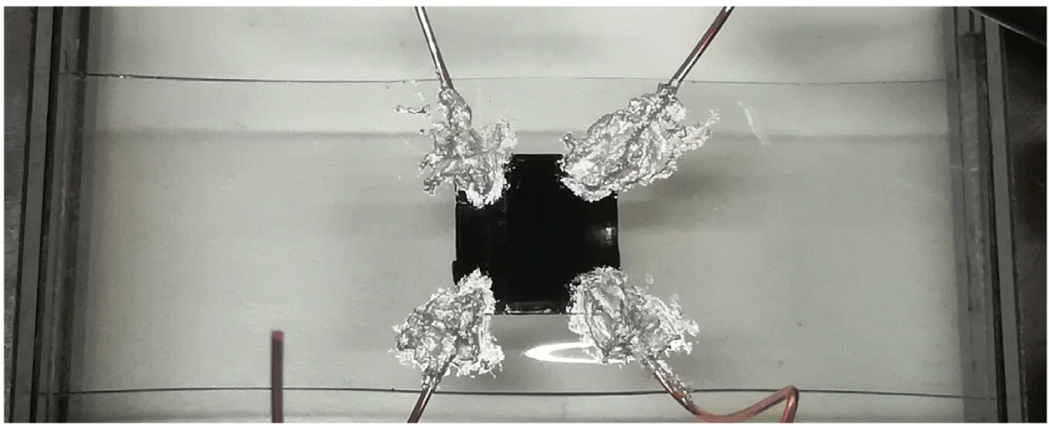

Fig. 2. Appearance of a test sample.

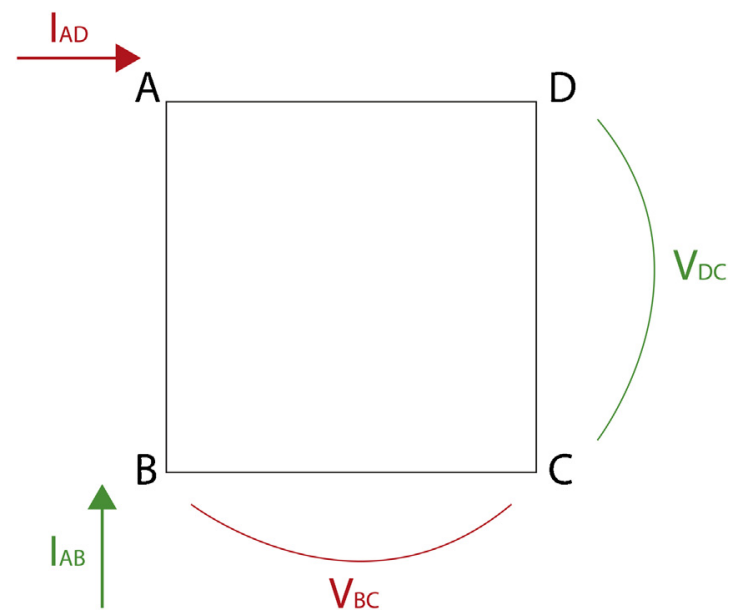

Fig. 3. Schematics of current (I) injection and voltage (V) measurement in the CNT square patch.

where $d=50 \mu \mathrm{m}$ is the average thickness of CNT patch, $R_{A B, C D}$ and $R_{B C, D A}$ result from $V_{D C} / I_{A B}$ and $V_{B C} / I_{A D}$ values, respectively, $\rho_{x}$ and $\rho_{y}$ are the resistivity in $\mathrm{X}$ - and Y-direction, respectively, and $\sqrt{\rho_{x} \rho_{y}}=R_{s} . d$, which implies that $R_{y y, \square} R_{x x, \square}=R_{S}{ }^{2}$.

$R_{S}$ is obtained from Van der Pauw relation (Eq. (4)).

$e^{-\pi R_{\text {vertical }} / R_{S}}+e^{-\pi R_{\text {horizontal }} / R_{S}}=1$

where $R_{\text {vertical }}$ and $R_{\text {horizontal }}$ are the means of the resistance experimental values obtained in the $\mathrm{Y}$ - and X-direction, respectively. Therefore, with $R_{y y, \square} / R_{x x, \square}$ and $R_{y y, \square} R_{x x, \square}$ values are obtained the sheet resistances in the two different directions, $R_{x x, \square}$ and $R_{y y, \square}$, respectively.

The electric anisotropy is defined as the electric resistance ratio $\left(R_{x x} / R_{y y}\right)$ between obtained resistances in transverse direction $(\mathrm{X})$, $R_{x x}$, and parallel direction (Y), $R_{y y}$, with respect to the CNT align- ment. These measurements were performed in static conditions and after deformation of the film substrates upon increased strain levels.

The gauge factor, GF, is defined in terms of the relative electrical resistance, $\Delta R / R_{0}$, to the mechanical strain, $\varepsilon$ :

$G F=\frac{\frac{\Delta R}{R_{0}}}{\varepsilon}$

\subsection{Electrical ressitivity versus strain measurements}

Furthermore, the electric anisotropy, $R_{x x} / R_{y y}$, the relative electric resistance $\left(R-R_{0} / R_{0}\right)$ and the GF, were obtained for samples knocked down onto PI, epoxy and PET films, which were then strained in directions $\mathrm{X}$ and $\mathrm{Y}$ (Fig. 4) with respect to CNT alignment direction. The samples were strained simultaneously in opposite directions using a manual microtester (Fig. 5), and the elongation of the samples $(\Delta l)$ were measured by a digital calliper (Mitutoyo).

\section{Results}

\subsection{SEM analysis}

The CNT alignment before and after the knock down process was assessed by SEM analysis. The results showed a good vertical CNT alignment after growth, however the VA-CNT forests showed a nonconstant height between $0.6-1.2 \mathrm{~mm}$ (Fig. 6), due to the variability of the CVD process [10].

The SEM images after the CNT knock down showed a realignment of the CNT forest in direction of the knock down process and a clear segmentation of the sample (Fig. 7). In region A, closer to the wafer, the CNT horizontal realignment is observed, despite the non-complete reorientation ( $0^{\circ}$ with horizontal direction). In comparison, the zone B on Fig. 7, which is in contact with the polymeric film, is a more compact zone, probably due to the CNT squashing and/or buckling during the knock down process [10]. a)

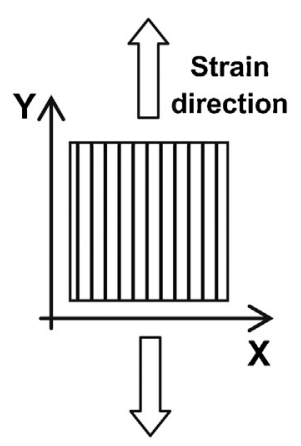

b)

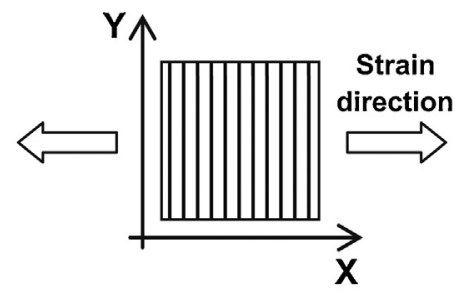

Fig. 4. Schematics of aligned CNT patch and strain directions: strain in CNT alignment ( $\left.\mathrm{Y}^{*}\right)$ direction (a) and in transverse direction (X) of CNT alignment (b). 


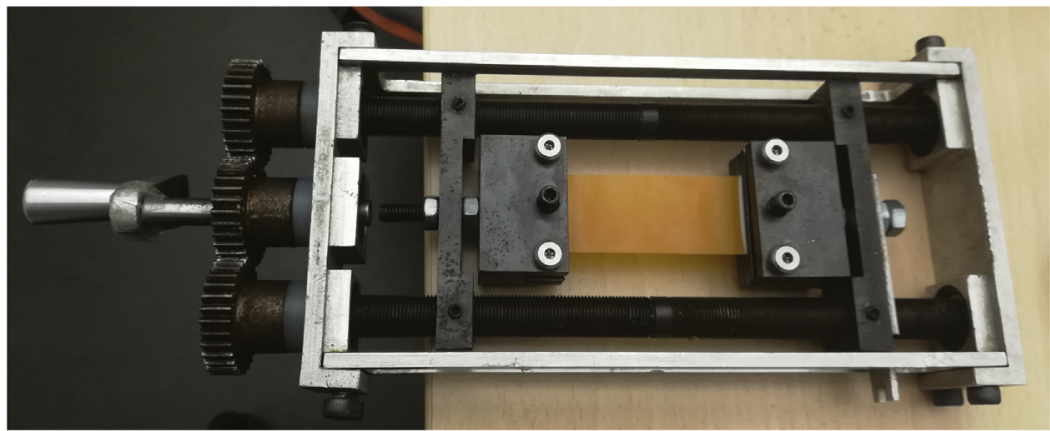

Fig. 5. Manual microtester used for the tensile test.
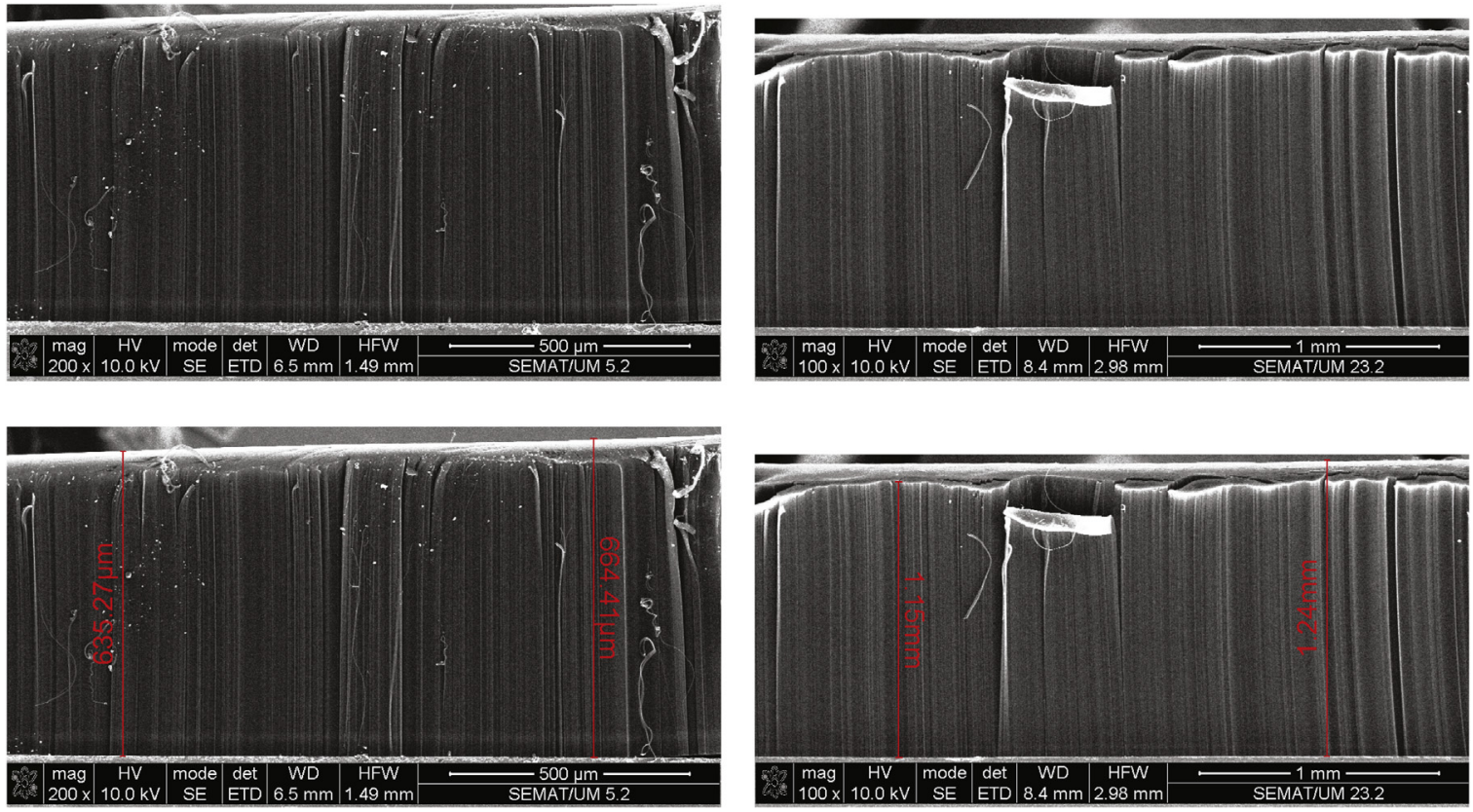

Fig. 6. SEM images of VA-CNT.
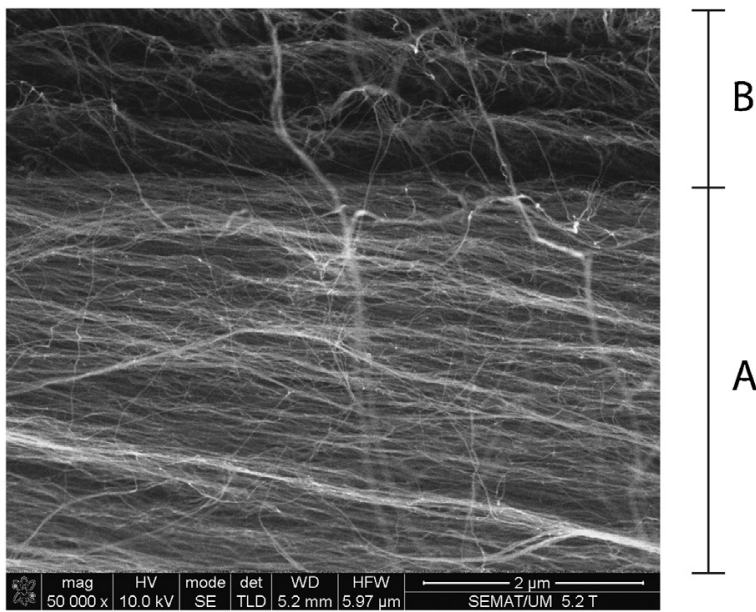

Fig. 7. SEM images of knocked down CNT: A) realignment of CNT and B) CNT compact zone.

\subsection{Raman spectroscopy}

As shown in Fig. 8, the Raman spectrum of the VA-CNT shows peaks at $1298 \mathrm{~cm}^{-1}$ (D-band), which is normally associated to defects on the CNT walls, and at $1549 \mathrm{~cm}^{-1}$ (G-band), which is asso-

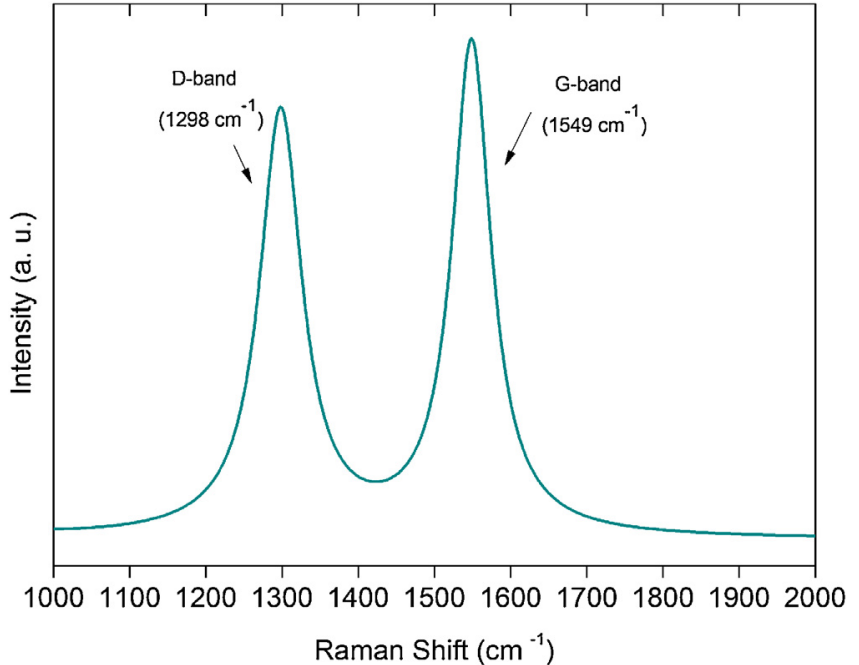

Fig. 8. Raman spectra of the VA-CNT.

ciated to the presence of crystalline graphite structures. The $A_{D} / A_{C}$ ratio calculated for the CNT forest is approximately 0.94 , which shows the good quality of the produced VA-CNT. 


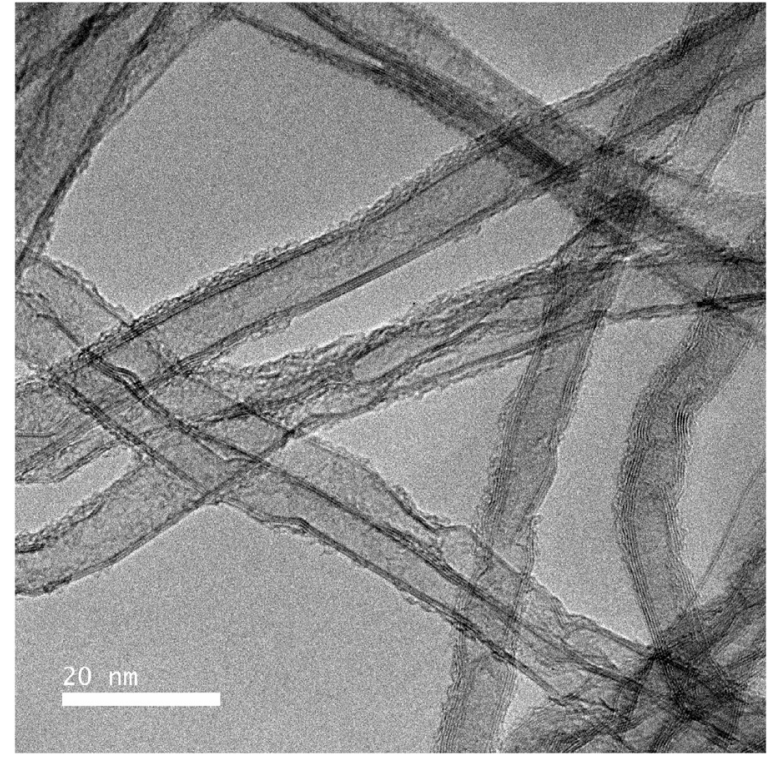

Fig. 9. TEM image of VA-CNT.

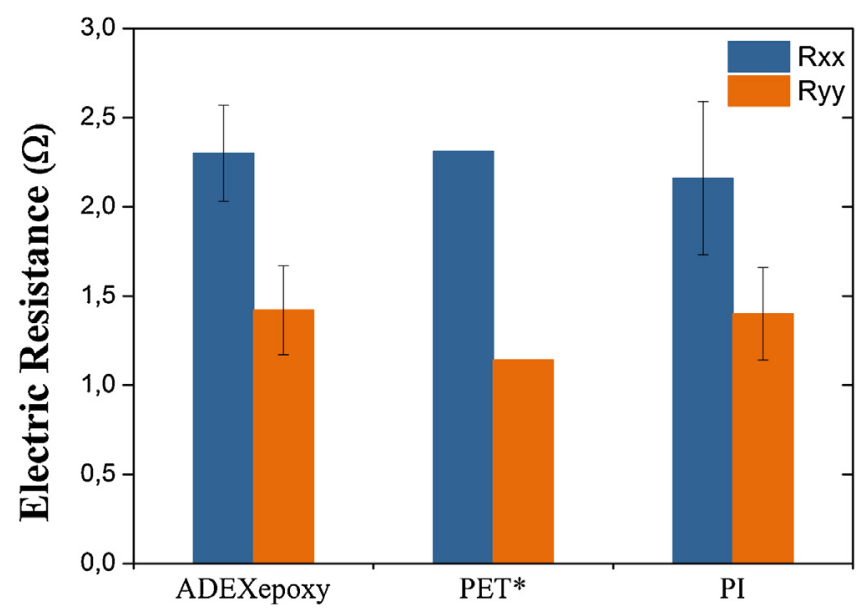

Fig. 10. Electric resistance results of $\mathrm{CNT} /$ polymeric films.

\subsection{TEM analysis}

TEM analysis was carried out to characterize the produced VACNT regarding their morphology. Therefore, their wall number and diameter were determined through Fiji software analysis of the TEM images (Fig. 9). A $t$-test was applied as statistic analysis to obtain confidence intervals, $\mathrm{CI}$, for the number of walls, inner and outer diameter, which were $(3.8,4.6),(3.17,4.17) \mathrm{nm}$ and $(4.55$, $5.76) \mathrm{nm}$, respectively, with a $95 \%$ confidence level.

\subsection{Electrical results in non-deformed state}

With no deformation, the obtained values of $R_{x x}$ and $R_{y y}$ are in order of the few Ohms (Fig. 10), being lower than the ones reported in literature [10,13]. As expected, $R_{x x}$ values are higher than $R_{y y}$. There is no significant variation of $R_{x x}$ and $R_{y y}$ upon the type of polymer substrate.

The electric anisotropy results in static mode are presented in Fig. $11 . R_{x x} / R_{y y}$ values are all higher than one, indicating that the electrical resistivity in the transverse direction of CNT alignment is higher. The values of electrical resistivity anisotropy range between 1.5-2.0, confirming the expected electrical anisotropy of aligned $\mathrm{CNT}$ patches. These results seem to be independent upon the type

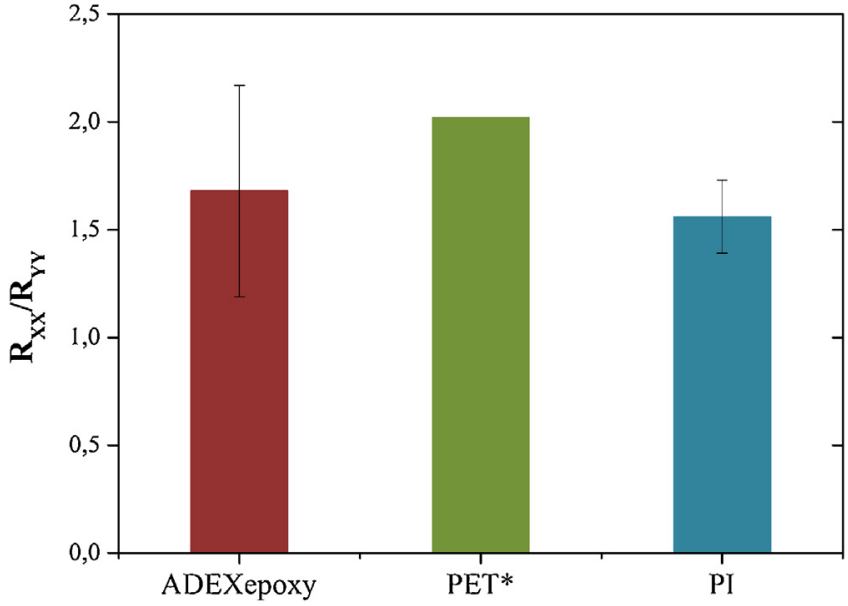

Fig. 11. Variability of anisotropy results obtained for $\mathrm{CNT} /$ polymeric films.

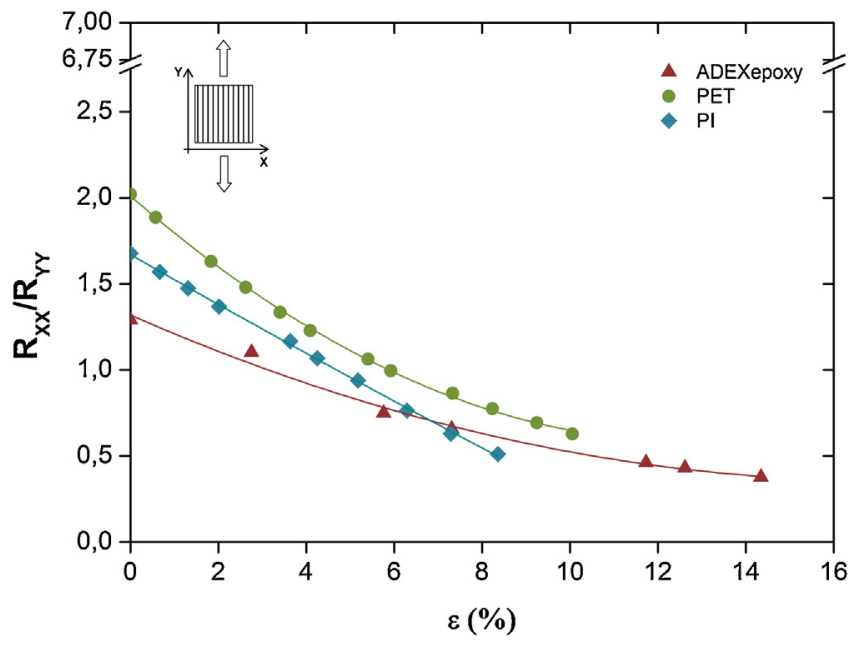

Fig. 12. Electrical resistivity anisotropy, $R_{x x} / R_{y y}$, versus strain, $\varepsilon$, obtained from the three different polymer substrates strained in direction of CNT alignment: CNT/epoxy, CNT/PET and CNT/PI.

of polymer substrate over which VA-CNT were knocked down. The variability of results may rise from the different CNT lengths [10] and from the manual knock down process (e.g., densification).

\subsection{Electrical resistivity results versus strain}

Fig. 12 shows the electrical anisotropy results at different strain levels for samples knocked down onto the three different polymer substrates. These results show that, the electrical resistivity anisotropy values always decrease with deformation increment, when the CNT patch is strained in the direction of its alignment (Y-direction). CNT/PI substrate sensors present the lowest strain limit (8-9\%), and conversely the CNT/epoxy substrate showed the highest strain values (15-16\%).

Fig. 13 shows the relative electrical resistance measurements at different strain levels for samples knocked down onto the three different polymer substrates. For these samples strained in direction of CNT alignment (Y-direction), the relative resistance in the strain direction increases with deformation increment, while in the opposite direction (X-direction) it decreases with a lower slope. This is an expected electric behaviour for samples strained along the direction of CNT alignment. Fig. 14a) shows a schematic of CNT conductive mechanism under strain when CNT are aligned with the strain direction. With the strain increment, the elec- 


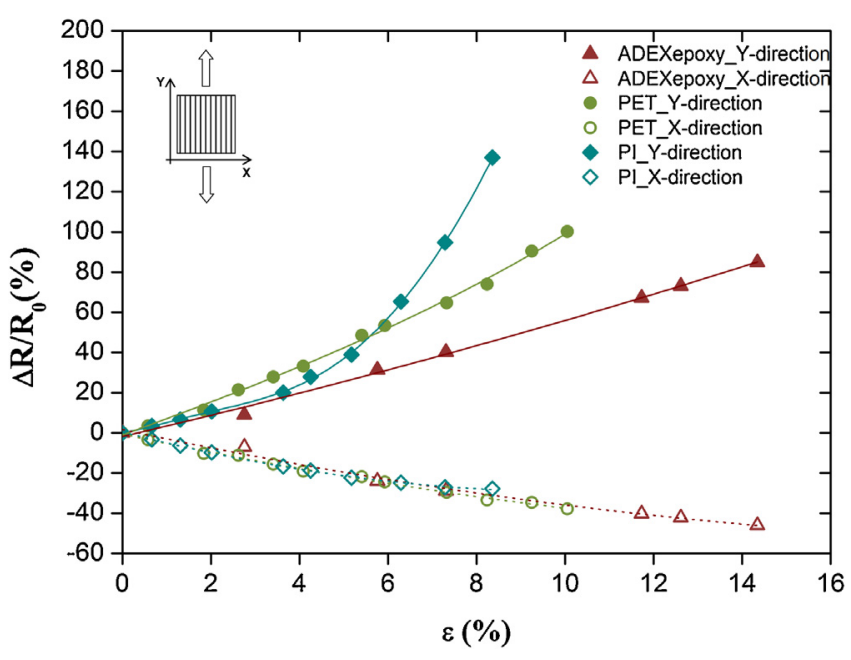

Fig. 13. Relative electrical resistance $\Delta R / R_{0}$ as function of the strain $\varepsilon$ for the three different polymer substrates strained in the direction of CNT alignment: CNT/epoxy, $\mathrm{CNT} / \mathrm{PET}$ and CNT/PI.

tron paths decrease, as the number of the CNT-CNT junctions that the electron has to tunnel throughout the CNT aligned network decreases, thus increasing sheet/tunnelling resistance in the Ydirection $[2,10,19]$. In the case of CNT/ADEX epoxy film, the relative resistance increases almost linearly with deformation increment, approximately from $31 \%$ to $85 \%$ for $6 \%$ and $14 \%$ deformation in the strain direction, respectively; while in the opposite direction there is a lower increment of approximately $16 \%$ to $36 \%$, for $6 \%$ and $15 \%$ deformation, respectively. This linear dependence anticipates a conduction mechanism based on the CNT-CNT junctions model [10]. The relative electrical resistance of CNT/PET sample increases almost linearly approximately from $11 \%$ to $74 \%$ for $1.87 \%$ and $8 \%$ deformation, respectively; while in the opposite direction there is a lower increase, approximately from $10 \%$ to $33 \%$ for $1.87 \%$ and $8 \%$ deformation, respectively. The relative resistance values of CNT/PI sample increases exponentially from $10 \%$ to $136 \%$ for $2 \%$ and $8 \%$ deformation, respectively, probably due to the CNT tunnelling conductive effect that dominates at higher deformations [3]. It is worth mentioning that the three polymeric films present different mechanical response upon an imposed strain, and this shall influence the deformation of the CNT network, and hence, its electrical response.

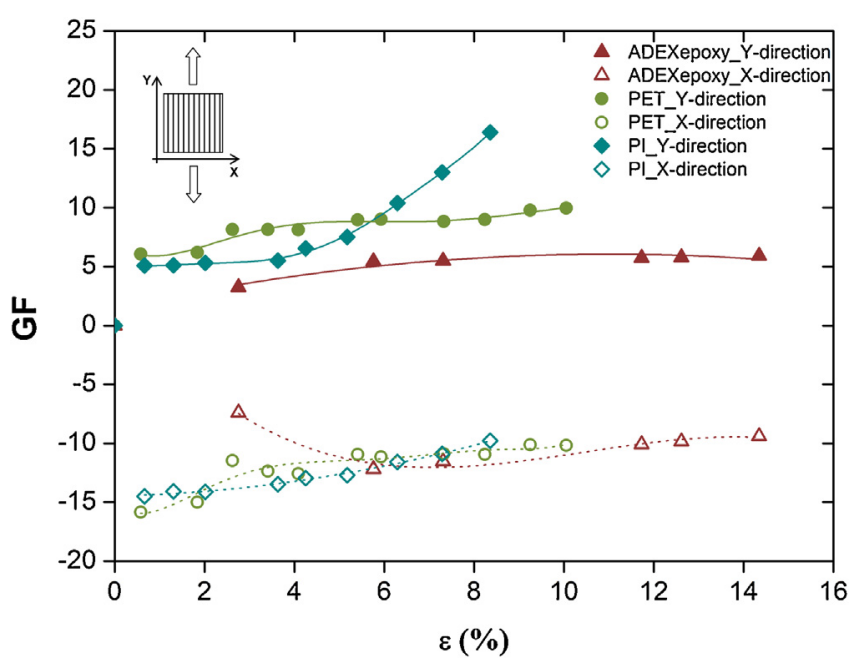

Fig. 15. Gauge factor (GF) versus strain $\varepsilon$ obtained from the three different samples strained in direction of CNT alignment: CNT/epoxy, CNT/PET and CNT/PI.

Fig. 15 shows the variations of the GF with the strain levels for samples knocked down onto the three different polymer substrates. The CNT/PET and CNT/PI samples show a step increase on GF over $0.5 \%$ of strain, followed by a low slope and than an exponential increase, while the CNT/epoxy sample shows an almost linear GF increase with strain. The CNT/PI sample shows higher GF values at higher deformations than the other samples. This is already anticipated in Fig. 13, where an exponentially increase on the relative electrical resistance is observed for this sample. In the transverse direction (X-direction), the GF values varied in a similar way. Regarding these strain sensitivity differences, it should be noted that the adhesion of the CNT to the polymeric films could have an important role on the CNT-CNT junction's deformation modes. In fact, a strong adhesion between the CNT patch and the polymeric film, with their similar thickness, can lead to mechanical reinforcement of the film affecting the CNT induced deformation and strain sensitivity. Specifically, the good adhesion of the CNT to the ADEX epoxy and PET films observed visually during the knock down process suggested that the CNT patch behaved as a mechanical film reinforcement, constricting the CNT slippage in the patch and therefore showing lower sensitivity compared to the $\mathrm{CNT} / \mathrm{PI}$ samples at higher deformations. Although the poor adhesion observed between CNT and PI films could mean higher slippage

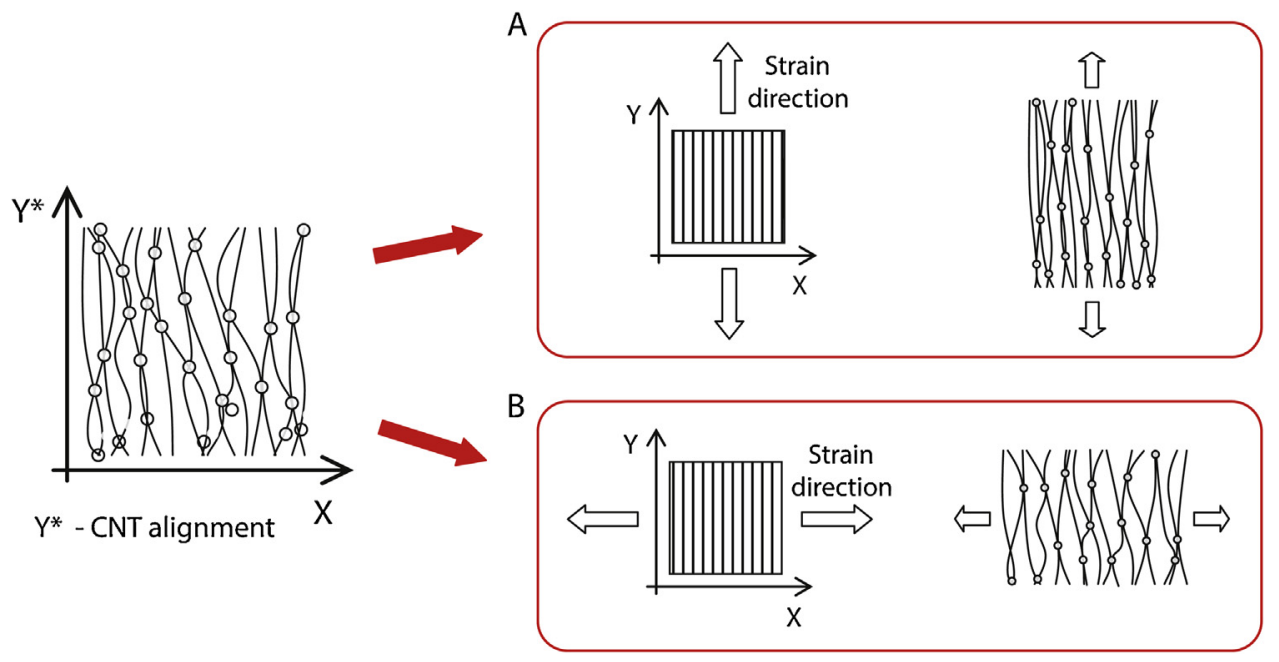

Fig. 14. Schematic of CNT conductive mechanism under strain in different directions: CNT alignment direction (A) and transverse direction of the alignment (B). 


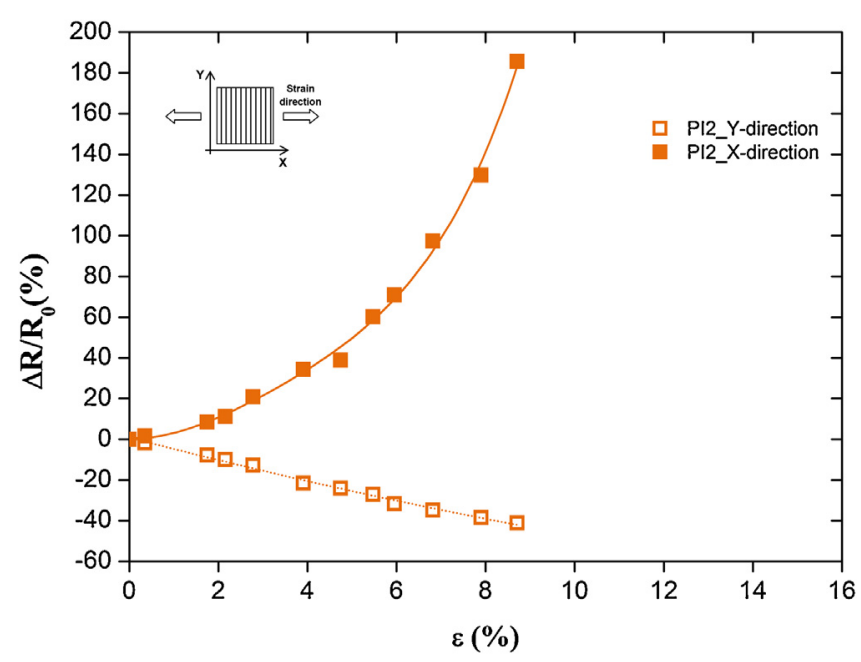

Fig. 16. Relative electrical resistance $\Delta R / R_{0}(\%)$ versus strain $\varepsilon(\%)$ obtained from the CNT/PI sample strained in transverse direction (X-direction) of CNT alignment (Y-direction).

between CNT in the patch, so higher sensitivity, and probably the strain was also not uniform along the full patch. It's worthy to mention that the better adhesion of the CNT to ADEXepoxy and PET films compared with the polyimide is reported elsewhere $[20,21]$.

Furthermore, all samples showed a high strain sensitivity even below $2 \%$ of deformation.

In addition, samples knocked down onto PI film were also strained transversely to CNT alignment (X-direction). As it can be seen in Fig. 16, the relative electrical resistance values increased exponentially until c.a. $130 \%$ at approximately $8 \%$ deformation in the strain direction, while in the opposite direction it decreased $38 \%$. This is an expected electric behaviour. As depicted in the schematic model of Fig. 14b), straining in transverse direction of CNT alignment affects the electron paths by decreasing the number of the CNT-CNT junctions that the electron has to tunnel throughout the CNT aligned network and thus, increasing sheet resistance in the X-direction $[2,10,19]$. This decrement of CNT-CNT junctions in X-direction, is accompanied by an increment on the number of these junctions on the opposed Y-direction, possible due to Poisson contraction effect on the CNT alignment direction. Again, there is a high strain sensitivity of the relative electrical resistance even for low strain values $2-3 \%$.

In Fig. 17, the electric resistance anisotropy values of the CNT/PI samples strained in X- and Y- directions are compared. As stated before, the applied strain affects the electron path in the CNT network, increasing CNT-CNT junction electrical resistance and the overall electric resistance in the strain direction. Therefore, the electric anisotropy decreases when the samples are strained in Y-direction, while it increases when strained in X-direction. The variations are much higher when samples are stretched in $\mathrm{X}$ direction, perpendicular to CNT alignment direction.

Moreover, the obtained GF values (Fig. 18) are higher and showed a slightly more sensitive response to deformation when the samples are strained transversely to CNT alignment direction (X-direction). This could suggest that, for the same strain level, the number of CNT-CNT junctions in X-direction has a higher decrement than on Y-direction.

For the same deformation level, the relative electrical resistance values of the produced A-CNT based sensors are higher than reported in the literature [3]. Despite having a slightly lower sensitivity at small deformations compared with others CNT based sensors [2], the production method reported here for CNT based sensor is much simpler and less time consuming.

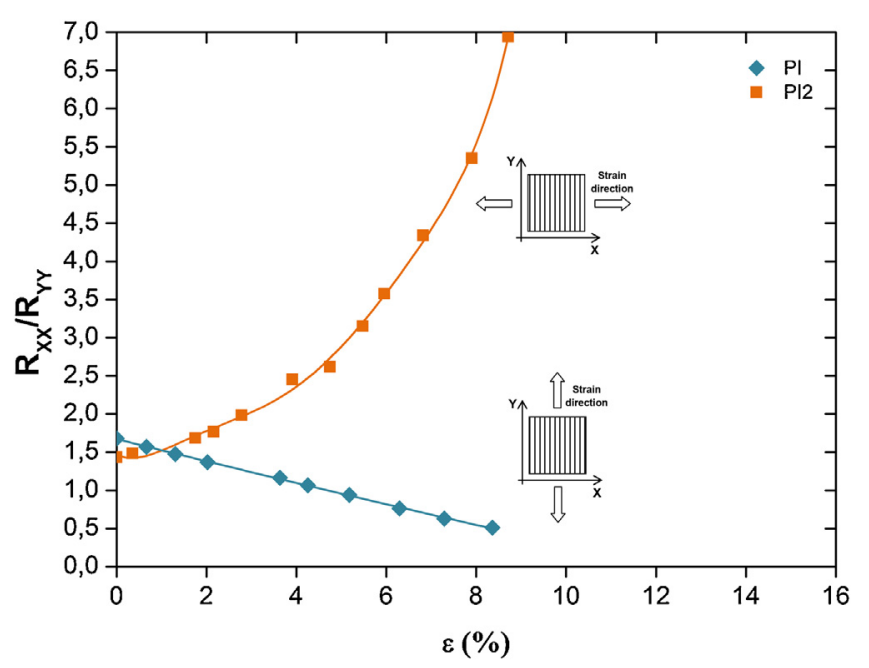

Fig. 17. Electric resistance anisotropy results of CNT/PI samples when strained in direction of CNT alignment (Y-direction) and transverse direction (X-direction), respectively.

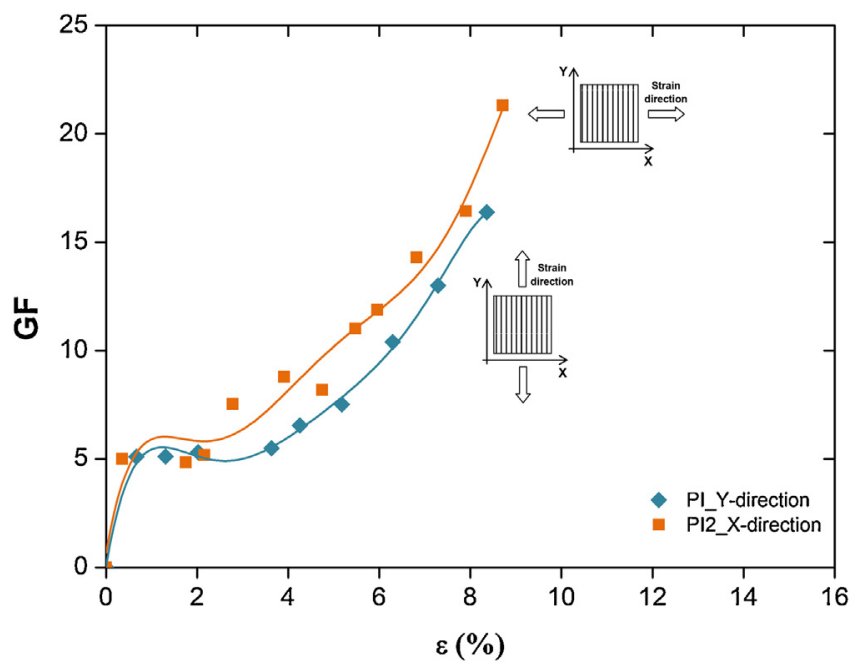

Fig. 18. Gauge factor results of CNT/PI samples when strained in the direction of CNT alignment (Y-direction) and in the transverse direction (X-direction).

\section{Conclusions}

An aligned CNT-based sensor for strain monitoring and sensing applications was presented. An apparatus for the CNT knock down process was manufactured, and also it was developed a MATLAB software for the resistance measurements in anisotropic materials.

The results of the electrical resistance anisotropy obtained in static mode (no stretching) from the different CNT/polymeric film samples suggest no influence of the substrate type, but a variability resultant from the CNT production process (e.g., CNT length and densification).

The relative electrical resistance and sensitivity to strain for the different samples show an increase with the deformation increment. These results are interpreted in terms of changes upon the number of CNT-CNT junctions with deformation, increasing the electrical resistivity. The adhesion between the CNT patch and the polymeric films is also determinant, i.e., a compromise between adhesion and slippage between CNT is crucial for the CNT patch sensitivity. Current aligned CNT-based sensors showed a high strain sensitivity even for strains lower than $2 \%$. In addition, the results of electrical resistivity sensitivity and anisotropy shows differences with the strain direction. The CNT patch strained in the transverse 
direction of CNT alignment showed slightly higher sensitivity compared with the ones strain in direction of CNT alignment. Moreover, the electric resistivity anisotropy values correspondent to these two strain directions present an opposite behaviour, which can be an important indicator of strain direction in a sensor.

\section{Acknowledgments}

This work was funded under the project "IAMAT - Introduction of advanced materials technologies into new product development for the mobility industries", with reference MITPTB/PFM/0005/2013, under the MIT-Portugal program exclusively financed by FCT - Fundação para a Ciência e Tecnologia. This work was carried out in part using the Advanced Electron Microscopy, Imaging and Spectroscopy Facility (TEM) of INL - Iberian Nanotechonology Laboratory.

\section{References}

[1] C.J. Shearer, A. Cherevan, D. Eder, Application and future challenges of functional nanocarbon hybrids, Adv. Mater. 26 (2014) 2296-2318, http://dx. doi.org/10.1002/adma.201305254.

[2] A. Bouhamed, C. Müller, S. Choura, O. Kanoun, Processing and characterization of MWCNTs / epoxy nanocomposites thin films for strain sensing applications, Sens. Actuators A Phys. 257 (2017) 65-72, http://dx.doi.org/10.1016/j.sna. 2017.01.022.

[3] N.T. Selvan, S.B. Eshwaran, A. Das, K.W. Stöckelhuber, S. Wießner, Piezoresistive natural rubber-multiwall carbon nanotube nanocomposite for sensor applications, Sens. Actuators A Phys. 239 (2016) 102-113, http://dx. doi.org/10.1016/j.sna.2016.01.004.

[4] M.D. Rein, O. Breuer, H.D. Wagner, Sensors and sensitivity: carbon nanotube buckypaper films as strain sensing devices, Compos. Sci. Technol. 71 (2011) 373-381, http://dx.doi.org/10.1016/j.compscitech.2010.12.008.

[5] L. Zhang, G. Zhang, C. Liu, S. Fan, High-density carbon nanotube buckypapers with superior transport and mechanical properties, Nano Lett. 12 (2012) 4848-4852.

[6] A. Li, A.E. Bogdanovich, P.D. Bradford, Aligned carbon nanotube sheet piezoresistive strain sensors, Smart Mater. Struct. 24 (2015), 095004, http:// dx.doi.org/10.1088/0964-1726/24/9/095004.

[7] B.E.J. García, A.J. Hart, B.L. Wardle, A.H. Slocum, Fabrication and nanocompression testing of aligned carbon-nanotube - polymer nanocomposites, Adv. Mater 19 (2007) 2151-2156, http://dx.doi.org/10.1002/ adma.200700237.

[8] O. Kanoun, C. Müller, A. Benchirouf, A. Sanli, T.N. Dinh, A. Al-Hamry, L. Bu, C. Gerlach, A. Bouhamed, Flexible carbon nanotube films for high performance strain sensors, Sensors 14 (2014) 10042-10071, http://dx.doi.org/10.3390/ S140610042.

[9] S. Roy, V. Jain, R. Bajpai, P. Ghosh, A.S. Pente, B.P. Singh, D.S. Misra, Formation of carbon nanotube bucky paper and feasibility study for filtration at the nano and molecular scale, J. Phys. Chem. C 116 (2012) 19025-19031, http://dx.doi. org/10.1021/jp305677h.

[10] J. Lee, I.Y. Stein, M.E. Devoe, D.J. Lewis, N. Lachman, S.S. Kessler, T. Samuel, Impact of carbon nanotube length on electron transport in aligned carbon nanotube networks, Appl. Phys. Lett. 106 (2015) 1-5, http://dx.doi.org/10. $1063 / 1.4907608$.

[11] G. Chen, D.N. Futaba, K. Hata, Interplay of wall number and diameter on the electrical conductivity of carbon nanotube thin films, Carbon N. Y. 67 (2014) 318-325, http://dx.doi.org/10.1016/j.carbon.2013.10.001.

[12] A.J. Hart, A.H. Slocum, Rapid growth and flow-mediated nucleation of millimeter-scale aligned carbon nanotube structures from a thin-film catalyst, J. Phys. Chem. B 110 (2006) 8250-8257.

[13] J. Lee, I.Y. Stein, S.S. Kessler, B.L. Wardle, Aligned carbon nanotube film enables thermally induced state transformations in layered polymeric materials, ACS Appl. Mater. Interfaces 7 (2015) 8900-8905, http://dx.doi.org/ 10.1021/acsami.5b01544.

[14] J.J. Stahl, A.E. Bogdanovich, P.D. Bradford, Carbon nanotube shear-pressed sheet interleaves for Mode i interlaminar fracture toughness enhancement, Compos. Part A Appl. Sci. Manuf. 80 (2016) 127-137, http://dx.doi.org/10. 1016/j.compositesa.2015.10.014.

[15] C.A. Coelho, L.A. Rocha, A.F. Silva, Carbon nanotubes - the challenges of the first syntheses trials, Int. Conf. Biomed. Electron. Devices (2015) 95-102, http://dx.doi.org/10.5220/0005201000950102.
[16] Y.W. Song, S.H. Park, W.S. Han, J.M. Hong, H.S. Kim, Single-step high-speed nanogranulation of metal alloy around carbon nanotubes by flash light irradiation, Mater. Lett. 65 (2011) 2510-2513, http://dx.doi.org/10.1016/j. matlet.2011.04.050.

[17] J.D. Wasscher, Note on four-point resistivity measurements on anisotropic conductors, Reports Philips Res. 16 (1961) 301-306.

[18] I. Kazani, G. De Mey, C. Hertleer, J. Banaszczyk, A. Schwarz, G. Guxho, L. Van Langenhove, Van Der Pauw method for measuring resistivities of anisotropic layers printed on textile substrates, Text. Res. J. 81 (2011) 2117-2124, http:// dx.doi.org/10.1177/0040517511416280.

[19] N. Hu, Y. Karube, M. Arai, T. Watanabe, C. Yan, Investigation on sensitivity of a polymer / carbon nanotube composite strain sensor, Carbon N. Y. 48 (2009) 680-687, http://dx.doi.org/10.1016/j.carbon.2009.10.012.

[20] M. xiang Jing, C. Han, M. Li, X. qian Shen, High performance of carbon nanotubes/silver nanowires-PET hybrid flexible transparent conductive films via facile pressing-transfer technique, Nanoscale Res. Lett. 9 (2014) 1-7, http://dx.doi.org/10.1186/1556-276X-9-588.

[21] M.C. Strus, C.I. Cano, R. Byron Pipes, C.V. Nguyen, A. Raman, Interfacial energy between carbon nanotubes and polymers measured from nanoscale peel tests in the atomic force microscope, Compos. Sci. Technol. 69 (2009) 1580-1586, http://dx.doi.org/10.1016/j.compscitech.2009.02.026.

\section{Biographies}

Ana Santos received the MSc degree in Biomedical Engineering at FCT/UNL, Portugal, in 2016. Her master degree dissertation was on biomaterials area: D̈evelopment of conductive membranes based on chitosan and reduced graphene oxide for biomedical applications: Presently, she is a researcher in IAMAT (Introduction of Advanced Materials Technologies into New Product Development for the Mobility Industries) project at UMinho, Portugal, and her work is focus on develop a CNT-based strain sensor

Luís Amorim is a researcher and a PhD candidate, in Institute of Polymers and Composites at University of Minho, Portugal. He obtained, in 2009, the MSc degree in Polymer Engineering at University of Minho on thermoplastic composites. After two years as extrusion process engineer, joined a research team to develop composites pressure vessels for commercial applications. Now, as researcher in IAMAT (Introduction of Advanced Materials Technologies into New Product Development for the Mobility Industries) project, his work is focus on study and understand impact damage mechanisms in advanced composites for aeronautic applications in order to improve their resistance.

J.P. Nunes is Auxiliary Professor and Deputy Executive Director of the Polyme Engineering Department and Senior Researcher at the Institute for Polymers and Composites (IPC/I3N) from the Minho University. He has the PhD in Polymer Science and Engineering from Minho University (1998) and is being working with composites since 1982. He participated in several International/National R\&D projects and published 4 book chapters and more than 150 papers in international journals and conferences. He has 6 patents and 2 awards for the development of innovative composite products and was Director of Engineering at the company VIDROPOL from 2000 to 2015

Luís A. Rocha received the degree in electronics engineering from the University of Minho, in 2000, and the Ph.D. (cum laude) degree from the Delft University of Technology, in 2005. He has been a MEMS Engineer at ASML, The Netherlands, in 2005, and was an Assistant Professor at Faculty of Engineering, University of Porto from 2006 to 2008. Since 2008, he has been an Associate Professor at University of Minho. His research activities focus on the fabrication of microsensors based on polymer microtechnologies, and on the study and use of the nonlinear MEMS dynamic behavior for new sensor applications.

Alexandre F. Silva (MSc'07-PhD'11) graduated, in 2007, in Biomedical Engineering at UMinho, Braga, Portugal. He obtained in 2011 the PhD degree in Leaders for Technical Industries, at the same institution, over the MIT-Portugal Program within the Engineering Design and Advanced Manufacturing (EDAM) focus-area. Presently, he is an invited assistant professor for the MIT Portugal's EDAM focus-area at UMinho. His research is driven by innovation and product-oriented in several fields related to life-enhancement product development.

Júlio C. Viana (D’91-MSc.93-PhD'00) received the Degree in Polymer Engineering from UMinho, Portugal, in 1991, the MSc degree in Computer Aided Mechanical Engineering Design from Queens University, Belfast, UK, in 1993, and the PhD from UMinho (in collaboration with CEMEF/Ecole des Mines de Paris) in 2000. He has been an Associate Professor at Department of Polymer Engineering, UMinho, since 2008. He has authored or co-authored 13 book chapters, 105 articles in international refereed journals and holds five patents. His research interests include relationships between processing, morphology, mechanical properties of polymers, advanced polymeric material systems, smart materials, printed and flexible electronics. 